\title{
Right Ventricular Endocardial Segmentation in CMR Images using a Novel Inter-modality Statistical Shape Modelling Approach
}

\author{
Concetta Piazzese ${ }^{1,2,3}$, M. Chiara Carminati ${ }^{3,4}$, Rolf Krause ${ }^{3}$, Angelo Auricchio ${ }^{3}$, Lynn Weinert ${ }^{5}$, \\ Gloria Tamborini ${ }^{1}$, Mauro Pepi ${ }^{1}$, Roberto M Lang ${ }^{5}$, Enrico G Caiani ${ }^{1}$ \\ ${ }^{1}$ Dipartimento di Elettronica, Informazione e Bioingegneria, Politecnico di Milano, Italy \\ ${ }^{2}$ Centre for Computational Medicine in Cardiology, Lugano, Switzerland \\ ${ }^{3}$ Centro Cardiologico Monzino IRCCS, Milan, Italy \\ ${ }^{4}$ Paul Scherrer Institut, Villigen, Switzerland \\ ${ }^{5}$ Department of Cardiology, University of Chicago, IL, USA
}

\begin{abstract}
Statistical shape modelling (SSM) approaches have been proposed as a powerful tool to segment the left ventricle in cardiac magnetic resonance (CMR) images. Our aim was to extend this method to segment the $R V$ cavity in CMR images and validate it compared to the conventional gold-standard (GS) manual tracing.

A SSM of the RV was built using a database of 4347 intrinsically $3 D$ surfaces, extracted from transthoracic $3 D$ echocardiographic (3DE) images of 219 retrospective patients. The SSM was then scaled and deformed on the base of some features extracted, with different strategies, from each short-axis plane until a stable condition was reached. The proposed approach, tested on 14 patients, resulted in a high correlation $\left(r^{2}=0.97\right)$ and narrow limits of agreement $( \pm 17 \%$ error $)$ when comparing the semiautomatic volumes to the GS, confirming the accuracy of this approach in segmenting the $R V$ endocardium.
\end{abstract}

\section{Introduction}

The relationship between the right ventricular (RV) function and different cardiac diseases has been proved by numerous works [1]. Cardiac magnetic resonance (CMR) is increasingly used as a standard tool in the evaluation of RV for its non-invasiveness and the ability to provide high-quality functional and anatomical images in any anatomical orientation.

Quantification of global and regional RV function depends upon accurate delineation of the endocardial wall. However, manual tracing of 2D CMR short-axis (SAX) slices in the acquired stack is time-consuming, tedious, and characterized by inter-observer variability.

Statistical shape modelling (SSM) has become a popular solution for image segmentation and analysis. This model-based technique have been successfully applied to segment left ventricular (LV) endocardium and epicardium in CMR images [2] but few works used a model-based technique to detect the RV endocardium.

A potential limitation in previous SSM applications to segment the ventricles from CMR images arises from the fact that the 3D SSM was built from a training set of 2D manual tracings on CMR SAX slices (with anisotropic resolution) interpolated along the third dimension [3]. In this way, detailed anatomical information at RV basal and apical level cannot be included in the SSM.

To overcome this limitation, we recently proposed an inter-modality statistical modelling approach to detect the LV endocardium in which the SSM was built from a first database of about 3000 3D endocardial meshes extracted from transthoracic three-dimensional echocardiography (3DE) images [4] of 205 patients and applied to CMR SAX images of 12 consecutive patients.

Based on these results, we hypothesized that the same SSM workflow could be applied for segmenting the RV endocardium in CMR SAX images.

\section{Methods}

\subsection{Statistical shape model generation}

The imaging database used in this study for the RV model construction was retrospectively collected from a clinical cohort of 219 subjects with various pathologies (181 with normal RV function, 23 with mitral valve prolapse, 10 with dilated cardiomyopathy, 5 with congenital diseases) that underwent 3DE examination at the University of Chicago, IL, USA or at Centro Cardiologico Monzino, Milano, Italy. Each 3DE dataset 
was analysed for RV endocardial quantification by using commercial software (TomTec Image Arena, München, Germany) and a triangulated surface, defined by 642 vertices and 1280 faces, describing the RV endocardium was automatically exported for each frame in the cardiac cycle, for a total of 4347 3D RV endocardial surfaces.

For each extracted 3D surface, the coordinates of three anatomical landmarks, corresponding to the apex (AP), to the centre of the tricuspid valve (TV) and to the pulmonary valve (PV) centre were uniquely identified (Figure 1) and used to co-register each surface in respect to a randomly chosen reference surface. Scaling was not performed on purpose, to include this anatomical variability in the SSM.

In order to keep only the meaningful information contained in the database, principal component analysis (PCA) was performed, with 1926 principal components (PC) to describe the whole data variability (Figure 2).

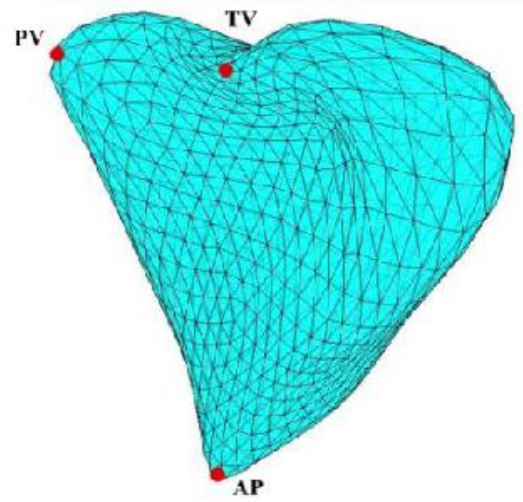

Figure 1. Exported RV surface mesh and three fiducial points corresponding to RV apex (AP), tricuspid valve centre (TV) and pulmonary valve (PV) centre.

\subsection{Pre-processing and user initialization}

Since the proposed SSM approach works on the whole 3D stack, in-plane correction for spatial misalignment was performed [5].

In order to properly scale and position the SSM inside the CMR data to analyse, four points (two points for TV leaflet insertion and one point for RV AP on the 4-ch long-axis (LAX) image and one point between the RV and the aorta (RA) on 3-ch LAX image) were initialized by the user.

\subsection{Image segmentation and model deformation}

The segmentation uses as input the initial scaled SSM and iteratively deforms it on the base of some features extracted from the stack of SAX CMR images, until a stable condition is reached. At each iteration, the statistical model was positioned inside the stack of SAX images to be segmented (Figure 3 a)) and the modelimage intersections were computed for each SAX plane (Figure 3 b))

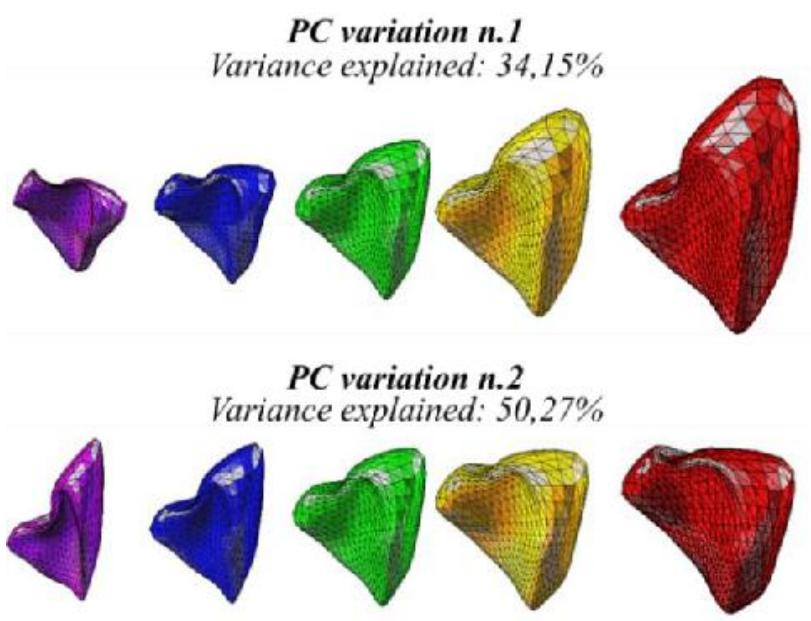

Figure 2. First two principal components (PC) and their possible variations constrained to $[-3 \sigma \div+3 \sigma]$, expressed as mean shape (green), $-3 \sigma$ (violet) $\div+3 \sigma$ (red), $-1.5 \sigma$ (blue) and $+1.5 \sigma$ (yellow).

The computed model-image intersections were used to explore the surrounding region. Due to the semilunar shape of the RV cavity on SAX images, the search space was investigated with two different strategies depending on the iteration number: at the first iteration, points lying on the line connecting each intersection with the RV geometric centre of all intersections were considered. For all the remaining iterations, pixel points were extracted from lines normal to the contour.

Pixel intensity profiles of the lines computed in the previous step were piled up to create a new 2D intensity image constituting the sampled research space (Figure 4 a)). The research space was subsequently processed by means of a k-means clustering approach using a different number of clusters depending on the position of each SAX plane.

Thresholding on blood clusters was then applied to define the endocardial interface, followed by morphological and derivative-based smoothing operations. The result was a binary image with white regions corresponding to $\mathrm{RV}$ blood pool and black regions corresponding to external structures. Consequently, the RV border interface was defined (Figure $4 \mathrm{c}$ )) as pixels corresponding to the transition from white to black and transformed back into the CMR reference system. 

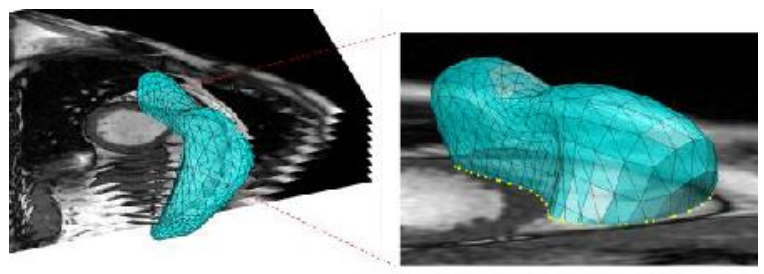

a)

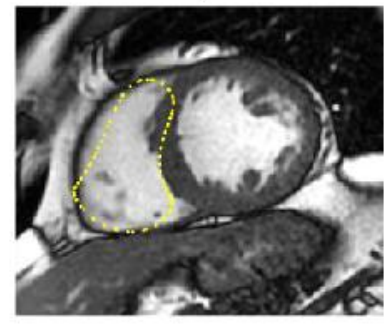

b)

Figure 3. Initial pose of the initial SSM inside the CMR data (a) and computation of model-image points as the intersections (yellow points) between the SSM and the SAX image (b).

Procrustes analysis [6] was applied to perform a rigid repositioning of the SSM model in the actual SAX stack (Figure 4 d)) and for each node a weighted displacement vector was computed. The SSM was then globally deformed to match the RV endocardial detected points in all SAX planes simultaneously. Model variations were controlled by restricting the shape deformations in the range $(-3 \sigma \div+3 \sigma)$ of the PCs selected during the statistical model creation.

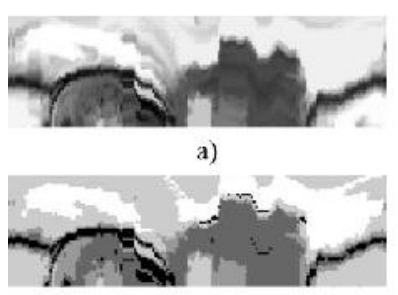

b)

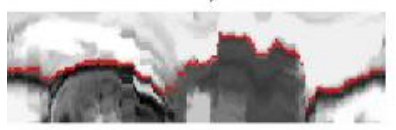

c)

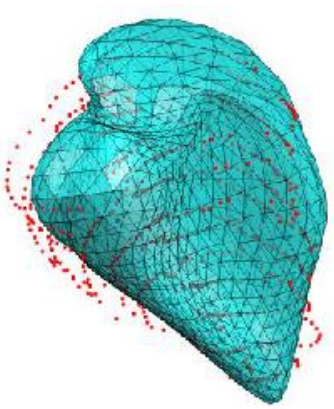

d)
Figure 4. RV endocardial segmentation steps and SSM deformation: a) grey-level image created by piling up the intensity profiles, b) k-means clustering, c) detected RV endocardial edge (red) superimposed on the initial greylevel image and d) detected endocardial points derived from the segmentation of the entire stack of SAX images (red), used to globally deform the statistical model.
The described steps were applied iteratively and when the convergence was reached, 3D RV endocardial volume was computed directly from the 3D mesh as the sum of the volume of each tetrahedron composing the 3D mesh.

\subsection{Imaging data and evaluation protocol}

The accuracy of the described SSM approach was tested by comparison with RV gold standard (GS) manual tracing on SAX CMR images at ED and ES phase of 14 patients ( 8 healthy, 3 with coronary artery disease and 3 with dilated cardiomyopathies).

Linear regression and Bland-Altman analyses were applied to compare the manual GS volumes and the semiautomated derived ones.

The point-to-surface distance was computed as the 3D distance from the points constituting the endocardial manual reference and the 3D model resulting from the SSM presented approach.

\section{Results}

The segmentation algorithm was implemented using Matlab (MathWorks, Natick, MA, USA) and it was successfully applied to detect RV endocardium in all selected patients. Processing time to analyse an entire stack of CMR SAX slices and obtain the resulting 3D RV surface was around 1 minute (including manual initialization) on a conventional laptop (IntelCore i7 @ 2.3 GHz, 16 GB RAM).

Figure 5 shows the resulting 3D surface superimposed on the 4-chamber LAX image in which it is possible to visually appreciate the correspondence of the deformed surface to the RV endocardial position as well as the visual anatomical correspondence at RV apex and base.

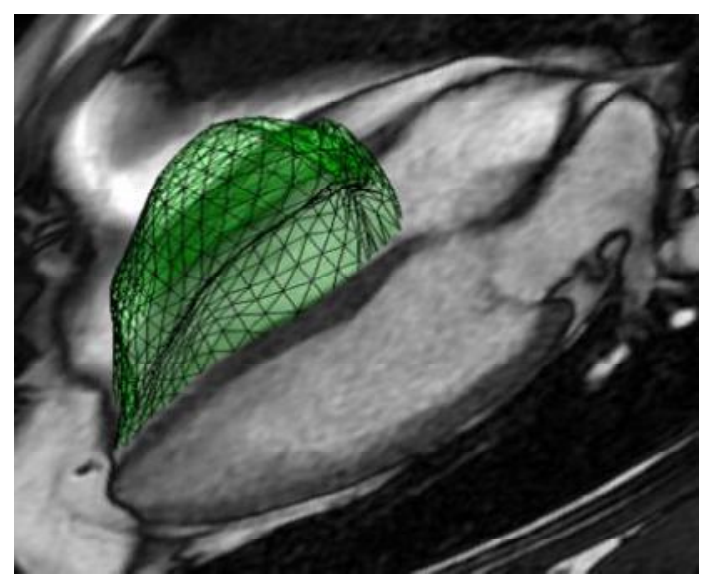

Figure 5. Resulting 3D surface mesh (green), describing the RV, superimposed on a 4-ch LAX image. 
Correlation analysis (Figure 6 left) between GS volumes and volumes computed directly from the 3D mesh showed a slope close to 1 and very high correlation coefficient (slope $=1.02, \mathrm{r}^{2}=0.97$ ). Bland-Altman analysis (Figure 6 right) resulted in a significant bias of $+9.3 \mathrm{ml}$ ) and narrow limits of agreement, equal to a $\pm 17 \%$ when compared to the GS.

A point-to-surface distance of $1.94 \pm 0.35 \mathrm{~mm}$ was found when evaluating the points constituting the RV endocardial manual reference and the 3D surface resulting from the proposed SSM method.
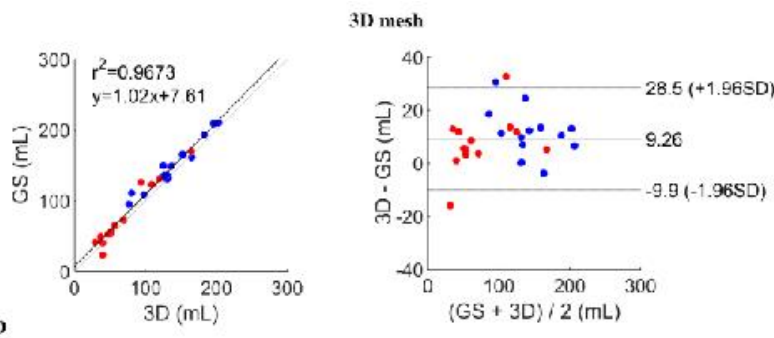

Figure 6. Linear correlation and Bland-Altman analyses between GS manually derived RV volumes and semiautomatic volumes computed directly from the 3D mesh

\section{Discussion}

This study proposed a new inter-modality SSM approach to segment the RV endocardium. To the best of our knowledge, this is the first time that a SSM trained on 3D RV endocardial surfaces obtained from 3DE images is applied to segment the RV cavity in SAX CMR images.

The use of 3D surfaces as training samples allowed obtaining a SSM consistent with RV anatomy, especially in the representation of $\mathrm{RV}$ apex and at the level of the tricuspid valve plane.

The proposed SSM approach showed to be accurate in segmenting the RV endocardium in CMR SAX images and required minimal user interaction for estimating the initial pose of the statistical model inside the stack of CMR SAX images. The output of the RV endocardial segmentation with the SSM approach is provided both as 2D RV contours and as a morphologically precise 3D RV endocardial surface mesh, in a representation that can be easily integrated into a patient-specific finite element model for surgical planning or cardiac electromechanical simulations.

Comparison analysis with the conventional GS showed a good correlation in RV volumes. An overestimation of LV volumes was evident when comparing the GS volumes to the volumes derived from $3 \mathrm{D}$ deformed mesh. This trend could be explained by the impact in the volume computation of LV basal and apical levels more defined in the 3D mesh and not completely visible when calculating volumes with the 2D method of discs, used as GS.

\section{Conclusion}

A new inter-modality SSM approach to segment the $\mathrm{RV}$ endocardium was proposed. The SSM trained on 3D RV endocardial surfaces extracted from 3DE images allowed accurate segmentation of the RV cavity in CMR images, resulting in a realistic RV morphology with an accurate anatomical delineation of both the apex and the base of the RV that could be utilized also for patientspecific modelling purposes.

\section{Acknowledgements}

This work was supported by the Italian Space Agency (contract number 2013-032-R.0, recepient E.G.C.).

\section{References}

[1] Matthews JC, Dardas TF, Dorsch MP, Aaronson KD. Right-sided heart failure: diagnosis and treatment strategies. Curr Treat Options Cardiovasc Med. 2008;10(4):329-41.3

[2] Heimann T, Meinzer HP. Statistical Shape Models for 3D Medical Image Segmentation: A Review. Medical Image Analysis 2009; 13: 543-563.

[3] Boehler T, Boskamp T, Mueller H, Hennemuth A, Peitgen HO. Evaluation of Active Appearance Models for Cardiac MRI. In Proc. BVM, pages 171-175. T. Tolxdorff, 2006.

[4] Caiani EG, Colombo A, Pepi M, Piazzese C, Maffessanti F, Lang RM, Carminati MC. 3D Left ventricular segmentation from MRI images for patient-specific modeling purposes. Europace 2014;16(suppl_4):iv96-101iv

[5] Carminati MC, Maffessanti F, Caiani EG. Nearly automated motion artifacts correction between multi breathhold short-axis and long-axis cine CMR images. Comput Biol Med 2014;46:42-50.

[6] Gower J. Generalized Procrustes analysis. Psychometrika 1975;40:33-51.

\section{Address for correspondence:}

Concetta Piazzese

Dipartimento di Elettronica, Informazione e Bioingegneria

Politecnico di Milano, Piazza L. da Vinci 32, Milano, Italy

E-mail: concetta.piazzese@ polimi.it 